\title{
Assessing and Management of Neurotoxicity After CAR-T Therapy in Diffuse Large B-Cell Lymphoma
}

\author{
Omar Castaneda-Puglianini $\mathbb{D}^{\prime}$ \\ Julio C Chavez ${ }^{2}$ \\ 'Virginia Commonwealth University, \\ Massey Cancer Center, Cellular \\ Immunotherapies and Transplant \\ Program, Richmond, VA, USA; \\ ${ }^{2}$ Department of Malignant Hematology, \\ Moffitt Cancer Center, Tampa, FL, USA
}

\begin{abstract}
Chimeric antigen receptor T-cell (CAR-T) therapy represents the most important advances in cancer immunotherapy, especially in hematological malignancies such as B-cell lymphomas. CAR-T cell therapy has significant activity in poor risk B-cell lymphomas. CAR-T cell therapy is associated with potentially life-threatening toxicities such as cytokine release syndrome (CRS) and neurotoxicity (NT). While CRS pathophysiology and management are well established, the understanding and treatment of NT continues to develop. All current CAR-T products approved for DLBCL have been associated with NT with some differences in their severity. As cell therapies continue to advance and its access broadening, it will be imperative for clinicians to be aware of the signs and symptoms of NT, its stratification and basic management.
\end{abstract}

Keywords: CAR-T, neurotoxicity, ICANS, encephalopathy

\section{Introduction}

Immunotherapy with chimeric antigen receptor T-cell (CAR-T) therapy is one of the most important advances in the treatment of cancer and, particularly, hematologic malignancies. In lymphoma, CAR-T cells targeting CD19 have been widely developed resulting in three commercially available products and several ongoing clinical trials. ${ }^{1}$ In diffuse large B-cell lymphoma (DLBCL), there are currently three CAR-T cell products approved (after at least 2 lines of therapy): axicabtagene ciloleucel (axi-cel), tisagenlecleucel (tisa-cel) and lisocabtagene ciloleucel (lisocel). Axi-cel, tisa-cel and liso-cel are genetically modified anti-CD19 autologous T-cells that are designed to target CD19 in B-cell malignancies. Based on the ZUMA-1, JULIET and TRANSCEND-NHL-001 studies, all three showed prominent activity in poor risk relapsed/refractory DLBCL with an overall response rate (ORR) between $50 \%$ and $82 \%$ with many patients having a durable response. ${ }^{2-5}$

The efficacy of CAR-T cell therapy is offset by potential class-effect toxicities that could be life-threatening and will require the implementation of appropriate measures to mitigate these side effects. CAR-T cell therapy is associated with high rates of unique toxicities, namely cytokine release syndrome (CRS) and neurotoxicity (NT). CRS is a well-known syndrome driven by a rapid release and expansion of inflammatory cytokines that leads to several systemic symptoms and clinical findings such as fevers, hypoxemia, constitutional symptoms, hypotension, tachycardia and organ dysfunction. ${ }^{6,7}$ The pathophysiology and management of CRS is well described and standardized, and involves the use of anti-cytokine therapy (ie, tocilizumab) with/without steroids. This treatment strategy leads to a rapid
Correspondence: Julio C Chavez Department of Malignant Hematology, Moffit Cancer Center, 12902 Magnolia Drive FOB, Tampa, FL, 33612, USA Email julio.c.chavez@moffitt.org 
resolution of symptoms. ${ }^{6-8}$ Unlike CRS, the understanding of NT pathophysiology and management is less established and continues to evolve. ${ }^{9}$

\section{Pathophysiology}

The pathophysiology of NT, currently known as immune effector cell-associated neurotoxicity syndrome (ICANS), continues to develop. Current knowledge highlights the disruption of the blood brain barrier (BBB) caused by inflammation and increased cytokines as a key initiating factors. ${ }^{10} \mathrm{BBB}$ disruption is triggered by endothelial cell activation as early event, which subsequently leads to BBB breakdown, increased permeability and coagulopathy as demonstrated in a mouse model. ${ }^{11}$ In healthy states, angiopoietin-1 (ANG1) and -2 (ANG2) remain in normal ratios, hence preventing endothelial activation. During severe ICANS, levels of ANG2 and ANG2: ANG1 ratio are significantly increased representing a disruption of the BBB and capillary leak. ${ }^{11}$ In addition, patients with severe ICANS had increased levels of pro-inflammatory cytokines such as tumor necrosis factor alpha (TNF-a), interferon gamma (IFN-g) and interleukin (IL)-6 that are likely released by CD14+ myeloid derived suppressor cells (MDSCs) which are also increased during NT. ${ }^{11,12}$ The presence of high levels of Von Willebrand Factor (WWF), high molecular weight WWF multimers and depleted ADAMTS 13 levels also supported the endothelial activated and coagulopathic state during severe ICANS. ${ }^{11}$

Other mouse models demonstrated the role of MDSCs and monocytes in NT pathophysiology and their key role as main sources of the above-mentioned pro-inflammatory cytokines including IL-1. ${ }^{13,14}$ Additionally, intratumoral interactions between CAR-T and macrophages/monocytes mediated by the CD40 ligand seems to be associated with CRS (and perhaps NT).$^{13}$ Administration of anakinra (an IL1 antagonist) prevented the development of CRS and NT in mouse models, without affecting the CAR-T cell efficacy. ${ }^{14}$

\section{Clinical Predictors and Biomarkers Clinical Predictors}

In general, neurological events have been reported more frequently and with more severity with axi-cel than tisa-cel and liso-cel, likely related to the type of costimulatory domain (CD28 vs 4-1BB). The pivotal ZUMA-1 study of axi-cel in R/R DLBCL, that included 111 patients, reported grade $\geq 3$ NT in $28 \%$ of patients. High tumor burden and CAR-T peak/expansion were associated with grade $\geq 3$ ICANS. $^{2,15}$ The frequency of grade $3 / 4$ NT in the JULIET trial (tisa-cel for R/R DLBCL) was 12\%, majority presenting with concurrent CRS. ${ }^{3}$ There were no specific factors associated with NT but appears that high tumor burden and elevated lactate dehydrogenase (LDH) were associated with severe NT. ${ }^{16}$ The TRANSCEND trial of liso-cel for R/R DLBCL reported grade $\geq 3$ NT in $10 \%$ of cases. Similar to the findings of ZUMA-1 and JULIET, patients with higher tumor burden (16\%), elevated LDH (19\%) and higher CAR expansion/peak were more likely to develop grade $\geq 3 \mathrm{NT}{ }^{5}$

In the off-trial setting, axi-cel had an incidence of grade $>3$ NT between $30 \%$ and $35 \%$. Bulky disease $(>10 \mathrm{~cm})$, higher day 0 and peak C-reactive protein (CRP) and peak ferritin levels were associated with severe NT. ${ }^{17,18}$ Similarly, higher baseline LDH levels and development of CRS were associated with NT severity. ${ }^{19}$ In another retrospective experience, the Massachusetts General Hospital reported that high CRP, LDH $\geq 400$ and thrombocytopenia were associated with grade $\geq 3 \mathrm{NT}^{20}$ Again, development of CRS was predictive of increased frequency and severity of NT, as previously seen in leukemias. ${ }^{20,21}$

In one of the largest datasets of neurotoxicity in lymphoma, the Dana Farber Cancer Institute developed a potential scoring system that could predict NT incidence and severity. This included age, LDH, baseline CRP, CRS (severity and timing), lymphoma histologic type, ferritin, leukocytes, number of doses of tocilizumab. A score of 6 or higher was predictive of grade 2 or higher NT. ${ }^{22}$

\section{Biomarkers and Cytokines}

Several cytokines have been implicated with the development and severity of ICANS in clinical studies. In patients with B-cell lymphoblastic leukemia treated with CAR-T cell therapy, the Memorial Sloan Kettering demonstrated that IL1a, IL-2, IL-3, IL-5, IL-6, IL-10, IL-15, IFN-g, G-CSF, GMCSF, and MCP1 were elevated and correlated with severe NT. ${ }^{23}$ The Fred Hutchinson Cancer and Research Center showed that increased levels of IL-6, IL-8, IFN-g, TNF-g and monocyte chemoattractant protein 1 (MCP-1) were associated with incidence and severity of neurotoxicity. ${ }^{24}$ The ZUMA-1 study showed that increased levels of IL-1Ra, IL2Ra, IL-6, IL-8, IL-10, IL-15, IFN-g, granzyme B, GM-CSF and ferritin were seen in patients with grade $>\mathrm{NT}^{2}$ The role of GM-CSF supporting inflammatory macrophages and monocytes generating CRS and NT has been established. ${ }^{12,25}$

In 75 patients who received axi-cel as standard of care for R/R LBCL at the Moffitt Cancer Center, point of care cytokine profiling was performed baseline (at the conditioning chemotherapy starting day and daily until 
discharge or up to 60 days post CAR-T cell infusion). ${ }^{26}$ Baseline levels of IL-6, ANG2, ANG2/ANG1 ratio and ferritin were associated with severe NT. Peak levels of IL6, IL-15, IFN-g, GM-CSF and ANG2/ANG1 were also seen in patients with grade $\geq 3 \mathrm{NT}^{26}$ Gene expression profiling by Nanostring in lymphoma tissue samples showed that low T-cell signature and high macrophage signature score were associated with NT severity, reflecting the role of the lymphoma microenvironment in the development of CAR-T related toxicities. ${ }^{26}$

\section{Clinical Findings}

ICANS occurs with a variable frequency and severity after CAR T cell therapy in DLBCL. It usually starts after the onset of CRS and, many times, after its resolution. Symptoms of ICANS can occur concomitantly with CRS, especially with severe grades of toxicities. ${ }^{22,27}$ Based on the published data of the 3 FDA approved CAR T-cell products for DLBCL, the median time to onset of neurotoxicity ranges from 5 to 9 days following CAR-T cell infusion; however, the incidence and clinical findings differ between patients receiving different products. ${ }^{2,3,5}$

Initial symptoms of ICANS do not have a specific pattern and can be vague. In most patients, early symptoms of ICANS include word-finding difficulty, reduced attention, calculation defect, tremors, impaired handwriting and lethargy. ${ }^{6,7}$ A clinical finding in the early phase of ICANS that seems to be very specific is expressive aphasia which can progress into global aphasia at later stages. ${ }^{24}$

Overall, neurotoxicity is, in general, self-limited. A summary of neurological events noted with approved CAR T-cell products for the treatment of DLBCL is shown in Table 1. Some symptoms and signs have been excluded from the definition of ICANS even though they may be associated with CAR T-cell therapy including headaches, hallucinations, asterixis, tremors and

Table I Neurological Events Noted with Approved CAR T-Cell Products for Large B-Cell Lymphoma

\begin{tabular}{|c|c|c|}
\hline Axicabtagene Ciloleucel & Lisocabtagene Maraleucel & Tisagenlecleucel \\
\hline $\begin{array}{l}\text { Encephalopathy: Includes encephalopathy, } \\
\text { cognitive disorder, confusional state, } \\
\text { depressed level of consciousness, disturbed } \\
\text { attention, hypersomnia, leukoencephalopathy, } \\
\text { memory impairment, mental status changes, } \\
\text { paranoia, somnolence, and stupor }\end{array}$ & $\begin{array}{l}\text { Encephalopathy: Includes encephalopathy, } \\
\text { confusional state, encephalopathy, mental status } \\
\text { changes, somnolence, lethargy, amnesia, } \\
\text { cognitive disorder, depressed level of } \\
\text { consciousness, memory impairment, flat affect, } \\
\text { depersonalization, disturbance in attention, } \\
\text { incoherent, and hypersomnia }\end{array}$ & $\begin{array}{l}\text { Encephalopathy: Includes encephalopathy, } \\
\text { cognitive disorder, confusional state, depressed } \\
\text { level of consciousness, disturbance in } \\
\text { attention, lethargy, mental status changes, } \\
\text { somnolence, and automatism }\end{array}$ \\
\hline $\begin{array}{l}\text { Delirium: Includes agitation, delirium, } \\
\text { disorientation, hallucination, hyperactivity, } \\
\text { irritability, restlessness, and delusion }\end{array}$ & $\begin{array}{l}\text { Delirium: Includes delirium, agitation, } \\
\text { disorientation, delusion, hallucination, visual } \\
\text { hallucination, and irritability }\end{array}$ & $\begin{array}{l}\text { Delirium: Includes delirium, agitation, } \\
\text { hallucination, hallucination visual, irritability, } \\
\text { and restlessness }\end{array}$ \\
\hline Headache & Headache: Includes headache and migraine & Headache: Includes headache and migraine \\
\hline $\begin{array}{l}\text { Dizziness: Includes dizziness, presyncope, } \\
\text { syncope }\end{array}$ & Dizziness: Includes dizziness and syncope & $\begin{array}{l}\text { Sleep disorder: Includes sleep disorder, } \\
\text { nightmares, and insomnia }\end{array}$ \\
\hline Aphasia: Includes aphasia, dysphasia & $\begin{array}{l}\text { Aphasia: Includes aphasia, dysarthria, dysphemia, } \\
\text { dysphonia, slow speech, and speech disorder }\end{array}$ & Anxiety \\
\hline Tremor & Tremor: Includes tremor and essential tremor & \\
\hline Ataxia & Ataxia: Includes ataxia and gait disturbance & \\
\hline $\begin{array}{l}\text { Motor dysfunction: Includes muscle } \\
\text { spasms, muscular weakness }\end{array}$ & $\begin{array}{l}\text { Cerebellar: Includes cerebellar syndrome, } \\
\text { dysmetria, balance disorder, dyskinesia and } \\
\text { impaired hand-eye coordination }\end{array}$ & \\
\hline \multicolumn{3}{|l|}{ Seizure } \\
\hline \multicolumn{3}{|l|}{ Dyscalculia } \\
\hline Myoclonus & & \\
\hline
\end{tabular}

Note: Data from references. ${ }^{3-6}$ 
myoclonus because they are usually treated symptomatically without triggering specific interventions such as corticosteroids. Also, headaches have been deemed not an appropriate indicator of ICANS leading to the exclusion from the definition as this is a non-specific symptom often seen in patients receiving chemotherapy or during febrile episodes in the absence of focal neurological deficits. ${ }^{7}$ Electroencephalographic typically shows diffuse generalized slowing with or without triphasic waves suggesting diffuse encephalopathy. ${ }^{28}$

Severe cases of ICANS with death have been described. Rapidly evolving cerebral edema and death from cerebral toxicity have been seen with anti-CD19 CAR T-cell and it was first described in the ROCKET study (JCAR015) where five patients developed fatal cerebral edema and death, which lead closure of the trial. $^{28-30}$ The incidence of fatal neurotoxicity following anti-CD19 CAR T-cell therapy has been reported to be $3 \%{ }^{11}$ As mentioned above, in LBCL, rates of severe NT in the pivotal trials ranged from $10 \%$ to $28 \%{ }^{2,3,5}$ It appears that the difference in the incidence of neurotoxicity may be accredited to the different costimulatory domains of the CAR T-cell constructs (CD28 or 4-1BB); however, no randomized trials have compared CAR T-cell constructs with different costimulatory domains in LBCL.

Radiological findings have been noted, specially, in severe NT, aside from the well-known cerebral edema, reported with JCAR015. ${ }^{30}$ Patients with severe NT had T2-FLAIR hyperintensities involving the brain stem, thalamus and corpus callosum that resolves with improvement of NT. ${ }^{24}$ Some acute changes with leptomeningeal enhancement, vasogenic edema and microscopic bleeding have been reported as well. ${ }^{11}$

\section{Grading and Stratification}

There were not well-developed and consensual systems to grade neurotoxicity that were available during the early phase of clinical trials with CAR-T cells in R/R DLBCL. Subsequent pivotal studies that lead to the approval of the currently commercially available CAR T-cell constructs adopted the National Institutes of Health (NIH) Common Terminology Criteria for Adverse Events (CTCAE).

In order to improve grading and surveillance of neurotoxicity, the CAR-T-cell-therapy-associated TOXicity (CARTOX) Working Group was formed, comprising investigators from different disciplines and multiple institutions to establish recommendations for monitoring, grading and management of CAR T-cell associated toxicities, this was called CARTOX-10 and included an assessment of the patient's orientation, naming, writing, and orientation (Table 2). ${ }^{28}$ Additionally, the CARTOX system developed the term CAR T-cellrelated encephalopathy syndrome (CRES) that included the evaluation of motor symptoms, seizures, and signs of elevated intracranial pressure (ICP) (Table 3). The ICP was evaluated by determination of cerebrospinal fluid (CSF) opening pressure and papilledema grading, according to the modified Frisén scale. ${ }^{28,31}$ CARTOX-10 was further refined giving birth to what is widely known as the immune effector cell-associated encephalopathy (ICE) score. The ICE score includes testing of simple commands, orientation, naming, writing, attention while removing the cumbersome, time consuming and potentially inaccurate evaluation of the ICP which may be not practical during daily practice.

Evidently, with the growing number of patients receiving adoptive cell therapy in particular CAR T-cell, there is a need to improve patient care and outcomes by allowing

Table 2 Encephalopathy Assessment Tools for Neurotoxicity Grading

\begin{tabular}{|l|l|}
\hline CARTOX-I0 & ICE \\
\hline $\begin{array}{l}\text { Orientation: Time (year, month), place (city, hospital), } \\
\text { president/prime minister of country of residence: } 5 \text { points }\end{array}$ & $\begin{array}{l}\text { Orientation: Time (year, month), place (city, hospital): } 4 \text { points } \\
\text { Naming: Ability to name } 3 \text { objects (eg, point to clock, pen, chair): } 3 \text { points } \\
\text { Waming: Ability to name three objects (eg, point to clock, pen, } \\
\text { Writing: Ability to write a standard sentence (eg, "our national bird is the bald } \\
\text { button): } 3 \text { points }\end{array}$ \\
$\begin{array}{l}\text { Writing: Ability to write a standard sentence (eg, "our } 1 \text { point } \\
\text { national bird is the bald eagle"): I point }\end{array}$ & $\begin{array}{l}\text { Following commands: Ability to follow simple commands (eg, "Show me } 2 \\
\text { fingers" or "Close your eyes and stick out your tongue"): I point }\end{array}$ \\
\hline Attention: Ability to count backwards from I00 by 10: I point & Total: 10 points \\
\hline Total: 10 points &
\end{tabular}

Notes: Data from references. ${ }^{6,8}$ Scoring: 10, no impairment; 7-9, grade I ICANS; 3-6, grade 2 ICANS; 0-2, grade 3 ICANS; 0 due to patient unarousable and unable to perform ICE assessment, grade 4 ICANS.

Abbreviations: CARTOX-10, CAR-T-cell-therapy-associated toxicity 10-point neurological assessment; ICE, immune effector cell-associated encephalopathy. 
Table 3 Grading of CAR T-Cell-Related Encephalopathy Syndrome (CRES)

\begin{tabular}{|l|l|l|l|l|}
\hline Symptom or Sign & Grade I & Grade 2 & Grade 3 & Grade 4 \\
\hline $\begin{array}{l}\text { Neurological } \\
\text { assessment score (by } \\
\text { CARTOX-10*) }\end{array}$ & $\begin{array}{l}\text { 7-9 (mild } \\
\text { impairment) }\end{array}$ & $\begin{array}{l}3-6 \text { (moderate } \\
\text { impairment) }\end{array}$ & 0-2 (severe impairment) & $\begin{array}{l}\text { Patient in critical condition, and/or obtunded } \\
\text { and cannot perform assessment of tasks }\end{array}$ \\
\hline $\begin{array}{l}\text { Raised intracranial } \\
\text { pressure }\end{array}$ & N/A & N/A & $\begin{array}{l}\text { Stage I-2 papilledema**, or CSF } \\
\text { opening pressure <20mmHg }\end{array}$ & $\begin{array}{l}\text { Stage 3-5 papilledema**, or CSF opening } \\
\text { pressure } \geq 20 \mathrm{mmHg} \text {, or cerebral edema }\end{array}$ \\
\hline $\begin{array}{l}\text { Seizures or motor } \\
\text { weakness }\end{array}$ & N/A & N/A & $\begin{array}{l}\text { Partial seizure, or non-convulsive } \\
\text { seizures on EEG with response to } \\
\text { BZD }\end{array}$ & $\begin{array}{l}\text { Generalized seizures, or convulsive or non- } \\
\text { convulsive status epilepticus, or new motor } \\
\text { weakness }\end{array}$ \\
\hline
\end{tabular}

Notes: Data from reference. ${ }^{8}$ As noted on Table 2; **Papilledema grading according to modified Frisén scale. ${ }^{12}$

Abbreviations: CAR, chimeric antigen receptor; CARTOX-I0, CAR-T-cell-therapy-associated toxicity I0-point neurological assessment; N/A, non-applicable; CSF, cerebrospinal fluid; EEG, electroencephalogram; BZD, benzodiazepine.

the standardized comparison of toxicities profiles and the efficacy of therapeutic interventions across studies and institutional practices under a common criterion. On that line, the American Society for Transplantation and Cellular Therapy (ASTCT) published a consensus grading system for CRS and ICANS which has been adopted as a systematic approach for reporting of CAR T-cell related toxicities. ${ }^{7}$ ICANS was defined as

A disorder characterized by a pathologic process involving the central nervous system following any immune therapy that results in the activation or engagement of endogenous or infused $\mathrm{T}$ cells and/or other immune effector cells. Symptoms or signs can be progressive and may include aphasia, altered level of consciousness, impairment of cognitive skills, motor weakness, seizures, and cerebral edema. ${ }^{7}$

In addition to the ICE score, ICANS grading also requires assessment of other neurological domains including level of consciousness, motor symptoms, seizures and signs of elevated ICP/cerebral edema with or in the absence of encephalopathy (Table 4).

It is clear that CTCAE is suboptimal for grading and assessment of neurotoxicity. As opposed to CTCAE v4.03,

Table 4 ASTCT Immune Effector Cell-Associated Neurotoxicity Syndrome (ICANS) Consensus Grading for Adults

\begin{tabular}{|c|c|c|c|c|}
\hline $\begin{array}{l}\text { Neurotoxicity } \\
\text { Domain }\end{array}$ & Grade I & Grade 2 & Grade 3 & Grade 4 \\
\hline ICE score*/** & $7-9$ & $3-6$ & $0-2$ & $\begin{array}{l}0 \text { (patient is unarousable and unable to perform } \\
\text { ICE) }\end{array}$ \\
\hline $\begin{array}{l}\text { Depressed level } \\
\text { of } \\
\text { consciousness }^{\dagger}\end{array}$ & $\begin{array}{l}\text { Awakens } \\
\text { spontaneously }\end{array}$ & $\begin{array}{l}\text { Awakens } \\
\text { to voice }\end{array}$ & Awakens only to tactile stimulus & $\begin{array}{l}\text { Patient is unarousable or requires vigorous or } \\
\text { repetitive tactile stimuli to arouse. Stupor or } \\
\text { coma }\end{array}$ \\
\hline Seizure & $\mathrm{N} / \mathrm{A}$ & $\mathrm{N} / \mathrm{A}$ & $\begin{array}{l}\text { Any clinical seizure focal or generalized that } \\
\text { resolves rapidly or nonconvulsive seizures } \\
\text { on EEG that resolve with intervention }\end{array}$ & $\begin{array}{l}\text { Life-threatening prolonged seizure ( }>5 \mathrm{~min} \text { ); or } \\
\text { Repetitive clinical or electrical seizures without } \\
\text { return to baseline in between }\end{array}$ \\
\hline Motor findings ${ }^{\ddagger}$ & $\mathrm{N} / \mathrm{A}$ & $\mathrm{N} / \mathrm{A}$ & N/A & $\begin{array}{l}\text { Deep focal motor weakness such as hemiparesis } \\
\text { or paraparesis }\end{array}$ \\
\hline $\begin{array}{l}\text { Elevated ICP/ } \\
\text { Cerebral edema }\end{array}$ & $\mathrm{N} / \mathrm{A}$ & $\mathrm{N} / \mathrm{A}$ & Focal/local edema on neuroimaging ${ }^{*}$ & $\begin{array}{l}\text { Diffuse cerebral edema on neuroimaging; } \\
\text { decerebrate or decorticate posturing; or cranial } \\
\text { nerve VI palsy; or papilledema; or Cushing's triad }\end{array}$ \\
\hline
\end{tabular}

Notes: Data from reference. ${ }^{8}$ ICANS grade is determined by the most severe event (ICE score, level of consciousness, seizure, motor findings, raised ICP/cerebral edema) not attributable to any other cause; for example, a patient with an ICE score of 3 who has generalized seizure is classified as grade 3 ICANS. *As noted on Table 2. **A patient with an ICE score of 0 may be classified as grade 3 ICANS if awake with global aphasia, but a patient with an ICE score of 0 may be classified as grade 4 ICANS if unarousable. †Depressed level of consciousness should be attributable to no other cause (eg, no sedating medication). $\ddagger$ Tremors and myoclonus associated with immune effector cell therapies may be graded according to CTCAE v5.0, but they do not influence ICANS grading. ¥Intracranial hemorrhage with or without associated edema is not considered a neurotoxicity feature and is excluded from ICANS grading. It may be graded according to CTCAE v5.0.

Abbreviation: N/A, not applicable. 
CAR T-Cell-Related Encephalopathy Syndrome (CRES) and American Society for Transplantation and Cellular Therapy (ASTCT) grading systems provide a more specific and accurate assessment of the occurrence and severity of neurotoxicity after CAR T-cell therapy as demonstrated by retrospective application of the three grading systems to the same patient data set from the JULIET trial. ${ }^{27,28}$

\section{Management}

The management of neurotoxicity post CAR T-cell therapy continues to evolve and constitutes an area of ongoing research. Different management algorithms have been published including consensus programs and institutionspecific interventions. ${ }^{2,3,28,32}$ Despite considerable variation in practice in terms of when and how to treat neurotoxicity, there are basic steps that guide most interventions. The CARTOX working group published one of the first general guidelines based on their extensive experience using axicabtagene ciloleucel for the management of CRS and neurotoxicity. ${ }^{28}$ The Society of Immunotherapy of Cancer (SITC) has recently released a clinical guideline for the management of CAR-T cell related complications including ICANS. ${ }^{8}$

In general, patients should have baseline neurological assessment prior to the infusion of CAR T-cell followed by close monitoring of new neurological signs/symptoms after CAR T-cell infusion. In most institutions, the baseline assessment includes a full neurological history and examination and a brain MRI to rule out any structural neurological abnormalities or possible central nervous involvement by lymphoma. Following CAR T-cell infusion, serial monitoring of ICE score, at least daily, is recommended (Table 3). ${ }^{6}$ Patients with presumed ICANS should undergo a detailed neurological examination and ICE scoring conducted by a medical provider experienced in the management of CAR-T cell patients. Evaluation by neurologist is encouraged, specially for severe cases. Neuroimaging is recommended, being a brain MRI with and without contrast the preferred modality if the clinical status of the patient allows it. ${ }^{28,33}$ For unstable and/or agitated patients, CT brain may be preferred. Neuroimaging of patients with ICANS usually show unremarkable findings for any structural pathology that explain neurotoxicity symptoms, even in patients with severe ICANS; however, it is needed to rule out other causes, such as intracranial hemorrhage or lymphoma involvement of the CNS. ${ }^{11,20,24,28}$ In some cases, patients with moderate to severe neurotoxicity might show patchy $\mathrm{T} 2$ hyperintensities throughout the white matter or T2 hyperintensities in the bilateral thalami, dorsal pons, and cerebral edema have been reported. . $^{211,24,28,30}$

Work up for patients with ICANS should also include some basic laboratory evaluation including CRP, CBC, CMP, fibrinogen, prothrombin time test, and international normalized ratio $(\mathrm{PT} / \mathrm{INR}){ }^{24}$ The CSF analysis as well as opening pressure should be pursued unless there is a contraindication. CSF should be assessed for chemistry, cytology, microbiology and virology. ${ }^{11,24,28,33}$ EEG is recommended even with lowgrade ICANS and in patients with seizures or suspected non-convulsive status epilepticus. Findings on EEG in patients with neurotoxicity, include diffuse slowing and frontal intermittent rhythmic delta activity, generalized background slowing and generalized periodic discharges which are both non-specific signs of diffuse cerebral dysfunction also seen in metabolic encephalopathy, infections, or centrally acting medications. ${ }^{11,24,34}$ Febrile patients require infectious workup with blood cultures, urine analysis, urine culture, chest X-ray, etc. per institutional guidelines.

The therapeutic strategy to follow is based on the grade of neurotoxicity and the coexistence of cytokine release syndrome. The treatment of neurotoxicity involves supportive care, frequent neurological monitoring and neuroimaging. ${ }^{7,8,35}$ Many institutions start prophylactic antiseizure therapy; however, the clinical evidence of benefit is not clear. The preferred antiseizure drug is levetiracetam, due to its minimal drug interactions. ${ }^{8}$ Previously, the negative impact of high-dose corticosteroids on the in vivo expansion of CAR T-cells and the presumed deleterious effect on the antileukemia control was described in a small cohort of patients with relapsed/refractory B cell acute lymphoblastic leukemia treated with autologous $\mathrm{T}$ cells expressing the $19-28 z$ CAR specific to the CD19 antigen. ${ }^{36}$ Most recently, in a retrospective analysis a higher cumulative dose and prolonged and early steroid use is associated with early progression as well as shorter overall survival after anti-CD19 CAR T-cell therapy in DLBCL patients. ${ }^{37}$ Regarding cytokine-directed therapy, tocilizumab is not usually recommended for treatment of neurotoxicity in the absence of concurrent CRS, because its inability to cross the blood-brain barrier and may paradoxically increase the concentration of IL-6 in the central nervous system. ${ }^{24}$ In the ZUMA-1 study, patients who received prophylactic tocilizumab on day 2 following axicabtagene ciloleucel infusion had a higher incidence of 
grade $\geq 3$ neurotoxicity per CTCAE v4.03 compared to patients that did not receive prophylactic tocilizumab. ${ }^{12}$ Data from preclinical models suggesting that anti-IL-1based therapies might provide a new therapeutic target for the management of neurotoxicity has led to the development of a significant number of clinical trials (NCT03430011, NCT04359784, NCT04148430, etc.). ${ }^{14}$ Since IL-1 seems a key mediator of neurotoxicity, the use of anakinra (an IL-1 receptor antagonist) could be a therapeutic strategy with potential efficacy. ${ }^{38}$ The group at MD Anderson Cancer Center has also reported the outcomes of a small cohort of patients receiving anakinra for the management of high-grade ICANS and HLH. In this series, 4 of 6 patients that received IL-1 blockade with anakinra for the management of high-grade ICANS experienced clinical benefit. ${ }^{39}$ Our approach for the management of ICANS is noted in Table 5.

\section{Future Strategies}

As the use of CAR-T therapy continues to expand for other conditions (multiple myeloma, acute leukemias, other B-cell malignancies, etc.), NT or ICANS will remain as one of the most important challenges. It also has the potential to add to the cost of care and physical burden for patient candidates for CAR-T cell therapy. ${ }^{19,40}$

A potential best strategy is the development of strategies to prevent NT. Given the preliminary efficacy of IL-1 blockade in improving NT in CAR-T treated patients, several trials using anakinra for NT prevention are ongoing (NCT04205838, NCT04359784, NCT04148430). In

Table 5 Management of ICANS

\begin{tabular}{|c|c|}
\hline $\begin{array}{l}\text { ASTCT ICANS } \\
\text { Grade }\end{array}$ & Management \\
\hline Grade I & $\begin{array}{l}\text {-Consider seizure prophylaxis with levetiracetam if not already started. } \\
\text {-Review of medications, avoid medications that can cause CNS depression. } \\
\text {-Swallowing assessment and aspiration precautions. } \\
\text {-Neurocognitive assessment Q6hrs using ICE scoring system. } \\
\text {-Neurology consult. } \\
\text {-Consider EEG. } \\
\text {-Consider lumbar puncture with opening pressure and samples for chemistry, cytology, virology, \& culture. } \\
\text {-Brain imaging (MRI preferred if no contraindication). Spinal MRI based on neurological findings. } \\
\text {-For febrile patients, infectious workup per institutional guidelines. } \\
\text {-Consider tocilizumab if concurrent CRS. }\end{array}$ \\
\hline Grade 2 & $\begin{array}{l}\text {-Supportive care and workup per Grade I. } \\
\text {-Consider dexamethasone IOmg IV every 6hrs or methylprednisolone equivalent. } \\
\text {-Tocilizumab if concurrent CRS. } \\
\text {-Consider transfer to intensive care unit. }\end{array}$ \\
\hline Grade 3 & $\begin{array}{l}\text {-Supportive care and workup per Grade I. } \\
\text {-Transfer to intensive care unit. } \\
\text {-Dexamethasone } 10-20 \mathrm{mg} \text { IV every } 6 \text { hours or methylprednisolone equivalent. } \\
\text {-High-dose methylprednisolone }(1000 \mathrm{mg} / \text { day) for focal/local edema. } \\
\text {-Seizure control with benzodiazepines (for short-term control) and levetiracetam } \pm \text { lacosamide. } \\
\text {-If evidence of increased ICP (stage I-2 by fundoscopy or opening pressure }>20 \mathrm{mmHg} \text { ), urgent neurology consultation to } \\
\text { guide management. } \\
\text {-Repeat neuroimaging if persistent grade } \geq 3 \text { ICANS. }\end{array}$ \\
\hline Grade 4 & $\begin{array}{l}\text {-Supportive care and workup per Grade I. } \\
\text {-Transfer to intensive care unit, may need mechanical ventilation for airway protection. } \\
\text {-High-dose methylprednisolone } 1000 \mathrm{mg} / \text { day for } 3 \text { days followed by taper. } \\
\text {-Seizure control per Grade } 3 \text {. } \\
\text {-Management of raised ICP per neurology/neurosurgery intensive care recommendations. May use hyperosmolar therapy } \\
\text { (mannitol/hypertonic saline), hyperventilation strategy. }\end{array}$ \\
\hline
\end{tabular}

Note: Data from references. ${ }^{6,8,17,18}$

Abbreviations: ASBMT, American Society for Transplantation and Cellular Therapy; ICANS, immune effector cell-associated neurotoxicity syndrome; CRS, cytokine release syndrome; EEG, electroencephalogram; ICE, Immune effector Cell-associated Encephalopathy; ICP, intracranial pressure; IV, intravenous. 
xenograft models of acute leukemia, the use of lenzilumab has shown that blocking GM-CSF, may reduce the incidence of CRS and NT without affecting CAR-T cell efficacy. $^{41}$ This is being investigated in the ZUMA-19 trial which combines lenzilumab with axi-cel for patients with R/R LBCL (NCT04314843). Finally, short course of steroids prophylactically may reduce the incidence of CRS and NT. The cohort 6 of the ZUMA-1, patients received dexamethasone $10 \mathrm{mg}$ on days 0 (prior to the infusion), 1 and $2 .^{42}$ While the rate of grade $\geq 3$ CRS was significantly decreased, grade $\geq 3$ NT rates did not change significantly but the incidence of NT within 72 hours from CAR-T cell infusion was lower. ${ }^{42}$ It is unclear whether this approach will be adopted widely; however, it may become attractive given its low cost and toxicity.

\section{Funding}

No funding source.

\section{Disclosure}

Dr Omar Castaneda Puglianini reports personal fees from Celgene Corporation, personal fees from Adaptive Biotechnologies Corporation, outside the submitted work. Dr Julio Chavez reports personal fees for consultancy from AbbVie, Morphosys, Kite/Gilead, Novartis, Karyopharm, ADC Therapeutics, TeneBio, and Janssen; and for Speaker Bureau from AstraZeneca, Epizyme, Genentech, Morphosys, BeGene, and BMS, a outside the submitted work. The authors report no other conflicts of interest in this work.

\section{References}

1. Brudno JN, Kochenderfer JN. Chimeric antigen receptor T-cell therapies for lymphoma. Nat Rev Clin Oncol. 2018;15(1):31-46. doi:10.1038/nrclinonc.2017.128

2. Neelapu SS, Locke FL, Bartlett NL, et al. Axicabtagene ciloleucel CAR T-cell therapy in refractory large B-cell lymphoma. $N$ Engl $J$ Med. 2017;377(26):2531-2544. doi:10.1056/NEJMoa1707447

3. Schuster SJ, Bishop MR, Tam CS, et al. Tisagenlecleucel in adult relapsed or refractory diffuse large B-cell lymphoma. $N$ Engl J Med. 2019;380(1):45-56. doi:10.1056/NEJMoa1804980

4. Locke Fl, Ghobadi A, Jacobson CA, et al. Long-term safety and activity of axicabtagene ciloleucel in refractory large B-cell lymphoma (ZUMA-1): a single-arm, multicentre, phase 1-2 trial. Lancet Oncol. 2019;20(1):31-42. doi:10.1016/S1470-2045(18)30864-7

5. Abramson JS, Palomba ML, Gordon LI, et al. Lisocabtagene maraleucel for patients with relapsed or refractory large B-cell lymphomas (TRANSCEND NHL 001): a multicentre seamless design study. Lancet. 2020;396(10254):839-852. doi:10.1016/S0140-6736(20)313 66-0

6. Lee DW, Gardner R, Porter DL, et al. Current concepts in the diagnosis and management of cytokine release syndrome. Blood. 2014;124 (2):188-195. doi:10.1182/blood-2014-05-552729
7. Lee DW, Santomasso BD, Locke FL, et al. ASTCT consensus grading for cytokine release syndrome and neurologic toxicity associated with immune effector cells. Biol Blood Marrow Transplant. 2019;25 (4):625-638. doi:10.1016/j.bbmt.2018.12.758

8. Maus MV, Alexander S, Bishop MR, et al. Society for Immunotherapy of Cancer (SITC) clinical practice guideline on immune effector cell-related adverse events. J Immunother Cancer. 2020;8(2):e001511. doi:10.1136/jitc-2020-001511

9. Acharya UH, Dhawale T, Yun S, et al. Management of cytokine release syndrome and neurotoxicity in chimeric antigen receptor (CAR) T cell therapy. Expert Rev Hematol. 2019;12(3):195-205. doi:10.1080/17474086.2019.1585238

10. Mackall CL, Miklos DB. CNS endothelial cell activation emerges as a driver of CAR T cell-associated neurotoxicity. Cancer Discov. 2017;7(12):1371-1373. doi:10.1158/2159-8290.CD-17-1084

11. Gust J, Hay KA, Hanafi LA, et al. Endothelial activation and bloodbrain barrier disruption in neurotoxicity after adoptive immunotherapy with CD19 CAR-T cells. Cancer Discov. 2017;7(12):1404-1419. doi:10.1158/2159-8290.CD-17-0698

12. Locke FL, Neelapu SS, Bartlett NL, et al. Preliminary results of prophylactic tocilizumab after axicabtageneciloleucel (axi-cel; KTEC19) treatment for patients with refractory, aggressive Non-Hodgkin Lymphoma (NHL). Blood. 2017;130(Supplement 1):1547.

13. Giavridis T, Van Der Stegen SJC, Eyquem J, Hamieh M, Piersigilli A, Sadelain M. CAR T cell-induced cytokine release syndrome is mediated by macrophages and abated by IL-1 blockade. Nat Med. 2018;24(6):731-738. doi:10.1038/s41591-018-0041-7

14. Norelli M, Camisa B, Barbiera G, et al. Monocyte-derived IL-1 and IL-6 are differentially required for cytokine-release syndrome and neurotoxicity due to CAR T cells. Nat Med. 2018;24(6):739-748. doi:10.1038/s41591-018-0036-4

15. Locke FL, Rossi JM, Neelapu SS, et al. Tumor burden, inflammation, and product attributes determine outcomes of axicabtagene ciloleucel in large B-cell lymphoma. Blood Adv. 2020;4(19):4898-4911. doi:10.1182/bloodadvances.2020002394

16. Maziarz RT, Schuster SJ, Ericson SG, et al. Cytokine release syndrome and neurotoxicity by baseline tumor burden in adults with relapsed or refractory diffuse large B-cell lymphoma treated with tisagenlecleucel. Hematol Oncol. 2019;37(S2):307. doi:10.1002/ hon.117_2630

17. Jacobson CA, Hunter BD, Redd R, et al. Axicabtagene ciloleucel in the non-trial setting: outcomes and correlates of response, resistance, and toxicity. J Clin Oncol. 2020;38(27):3095-3106. doi:10.1200/ JCO.19.02103

18. Nastoupil LJ, Jain MD, Feng L, et al. Standard-of-care axicabtagene ciloleucel for relapsed or refractory large B-cell lymphoma: results from the US lymphoma CAR T consortium. J Clin Oncol. 2020;38 (27):3119-3128. doi:10.1200/JCO.19.02104

19. Wudhikarn K, Pennisi M, Garcia-Recio M, et al. DLBCL patients treated with CD19 CAR T cells experience a high burden of organ toxicities but low nonrelapse mortality. Blood Adv. 2020;4(13):30243033. doi:10.1182/bloodadvances.2020001972

20. Karschnia P, Jordan JT, Forst DA, et al. Clinical presentation, management, and biomarkers of neurotoxicity after adoptive immunotherapy with CAR T cells. Blood. 2019;133(20):2212-2221. doi:10.1182/ blood-2018-12-893396

21. Gofshteyn JS, Shaw PA, Teachey DT, et al. Neurotoxicity after CTL019 in a pediatric and young adult cohort. Ann Neurol. 2018;84(4):537-546. doi:10.1002/ana.25315

22. Rubin DB, Al Jarrah A, Li K, et al. Clinical predictors of neurotoxicity after chimeric antigen receptor T-cell therapy. JAMA Neurol. 2020;77(12):1536-1542. doi:10.1001/jamaneurol.2020.2703

23. Hay KA, Hanafi LA, Li D, et al. Kinetics and biomarkers of severe cytokine release syndrome after CD19 chimeric antigen receptormodified T-cell therapy. Blood. 2017;130(21):2295-2306. doi:10.11 82/blood-2017-06-793141 
24. Santomasso BD, Park JH, Salloum D, et al. Clinical and biological correlates of neurotoxicity associated with CAR T-cell therapy in patients with B-cell acute lymphoblastic leukemia. Cancer Discov. 2018;8(8):958-971. doi:10.1158/2159-8290.CD-17-1319

25. Singh N, Hofmann TJ, Gershenson Z, et al. Monocyte lineagederived IL-6 does not affect chimeric antigen receptor T-cell function. Cytotherapy. 2017;19(7):867-880. doi:10.1016/j.jcyt.2017.04.001

26. Faramand R, Jain M, Staedtke V, et al. Tumor microenvironment composition and severe Cytokine Release Syndrome (CRS) influence toxicity in patients with large B-cell lymphoma treated with axicabtagene ciloleucel. Clin Cancer Res. 2020;26(18):4823-4831. doi:10.1158/1078-0432.CCR-20-1434

27. Maziarz RT, Schuster SJ, Romanov VV, et al. Grading of neurological toxicity in patients treated with tisagenlecleucel in the JULIET trial. Blood Adv. 2020;4(7):1440-1447. doi:10.1182/bloodadvances.2019001305

28. Neelapu SS, Tummala S, Kebriaei P, et al. Chimeric antigen receptor T-cell therapy - assessment and management of toxicities. Nat Rev Clin Oncol. 2018;15(1):47-62. doi:10.1038/nrclinonc.2017.148

29. Turtle CJ, Hay KA, Gust J, et al. Cytokine release syndrome (CRS) and neurotoxicity (NT) after CD19-specific chimeric antigen receptor- (CAR-) modified T cells. J Clin Oncol. 2017;35(15_suppl):3020. doi:10.1200/JCO.2017.35.15_suppl.3020

30. Poh A. JCAR015 in ALL: a root-cause investigation. Cancer Discov. 2018;8(1):4-5.

31. Frisen L. Swelling of the optic nerve head: a staging scheme. J Neurol Neurosurg Psychiatry. 1982;45(1):13-18. doi:10.1136/jnnp.45.1.13

32. Brudno JN, Kochenderfer JN. Toxicities of chimeric antigen receptor T cells: recognition and management. Blood. 2016;127(26):33213330. doi:10.1182/blood-2016-04-703751

33. Rubin DB, Danish HH, Ali AB, et al. Neurological toxicities associated with chimeric antigen receptor T-cell therapy. Brain. 2019;142 (5):1334-1348. doi:10.1093/brain/awz053
34. Herlopian A, Dietrich J, Abramson JS, Cole AJ, Westover MB. EEG findings in CAR T-cell therapy-related encephalopathy. Neurology. 2018;91(5):227-229. doi:10.1212/WNL.0000000000005910

35. Neelapu SS. Managing the toxicities of CAR T-cell therapy. Hematol Oncol. 2019;37(Suppl 1):48-52. doi:10.1002/hon.2595

36. Davila ML, Riviere I, Wang X, et al. Efficacy and toxicity management of $19-28 z$ CAR T cell therapy in B cell acute lymphoblastic leukemia. Sci Transl Med. 2014;6(224):224ra225. doi:10.1126/ scitranslmed.3008226

37. Strati P, Ahmed S, Furqan F, et al. Prognostic impact of corticosteroids on efficacy of chimeric antigen receptor T-cell therapy in large B-cell lymphoma. Blood. 2021;137(23):3272-3276. doi:10.1182/ blood.2020008865

38. Tummala S. Anakinara to mitigate CAR T cell therapy associated toxicity (4838); 2021.

39. Strati P, Ahmed S, Kebriaei P, et al. Clinical efficacy of anakinra to mitigate CAR T-cell therapy-associated toxicity in large B-cell lymphoma. Blood Adv. 2020;4(13):3123-3127. doi:10.1182/bloodadvances. 2020002328

40. Hernandez I, Prasad V, Gellad WF. Accounting for All costs in the total cost of chimeric antigen receptor T-cell immunotherapy-reply. JAMA Oncol. 2018;4(12):1785-1786. doi:10.1001/jamaoncol.2018. 4657

41. Sterner RM, Sakemura R, Cox MJ, et al. GM-CSF inhibition reduces cytokine release syndrome and neuroinflammation but enhances CAR-T cell function in xenografts. Blood. 2019;133(7):697-709. doi:10.1182/blood-2018-10-881722

42. Oluwole OO, Bouabdallah K, Muñoz J, et al. Prophylactic steroid use with Axicabtagene Ciloleucel (Axi-Cel) in Patients (Pts) with Relapsed/Refractory Large B Cell Lymphoma (R/R LBCL). Presented at: 2021 TCT $\mid$ Transplantation \& Cellular Therapy Meetings of ASTCT and CIBMTR.
Journal of Blood Medicine

\section{Publish your work in this journal}

The Journal of Blood Medicine is an international, peer-reviewed, open access, online journal publishing laboratory, experimental and clinical aspects of all aspect pertaining to blood based medicine including but not limited to: Transfusion Medicine; Blood collection, Donor issues, Transmittable diseases, and Blood banking logistics; Immunohematology; Artificial and alternative blood based therapeutics; Hematology; Biotechnology/nanotechnology of blood related medicine; Legal aspects of blood medicine; Historical perspectives. The manuscript management system is completely online and includes a very quick and fair peer-review system. Visit http://www.dovepress.com/testimonials.php to read real quotes from published authors. 